\section{Outpatient High-Dose Urokinase Infusion Improves Dialysis Catheter Longevity: A Prospective Observational Study}

Rajiv Saran, ${ }^{1,2}$ Vijaya Venkataraman, ${ }^{1}$ Sean F. Leavey, ${ }^{2}$ Ron Usovsky, ${ }^{1}$ Kumari Usha, ${ }^{1}$ Zbylut J. Twardowski ${ }^{1}$

University of Missouri-Columbia, ${ }^{1}$ Columbia, Missouri; University of Michigan, ${ }^{2}$ Ann Arbor, Michigan, U.S.A.
C atheter thrombosis is a major limiting factor affecting catheter survival in hemodialysis (HD). A previous retrospective study highlighted the utility of outpatient intradialytic high-dose urokinase (HDU) infusion in the treatment of HD catheter thrombosis. The present study was designed to prospectively evaluate the effectiveness of 250000 IU urokinase given as a 3-hour infusion during HD. The infusion would follow detection of a drop in blood flow thought to be secondary to thrombosis [a "thrombotic event" (TE)] after exclusion of nonthrombotic causes of drop in blood flow [a "nonthrombotic event" (NTE)], such as systemic hypotension or suboptimal position of the catheter on $x$ ray. An "event" was defined as a drop in pump blood flow by greater than or equal to $20 \%$ of the prescribed blood flow. Twenty patients (mean age 57.7 years; 13 males) with documented or potentially long-term catheters were recruited (total catheters used, 30) from an outpatient HD unit. A variety of catheter types were used. Catheter position was confirmed on $x$ ray. Hemodialysis sessions were monitored for 6 months or until catheter removal (whichever was earlier). Thrombotic and NTEs (mostly related to drop in blood flow) were noted. The use of intradialytic HDU infusions was monitored. Improvement was defined as reestablishment of blood flow to within $10 \%$ of prescription.

There were 24 TEs in 11 patients and 15 NTEs in 7 patients. Twenty-one of 22 (95\%) TEs responded to HDU, with complete restoration of blood flow; in 1/22 HDU-treated instances there was a partial response. For 2/24 TEs the patients underwent catheter stripping with good results. Twelve of 15 NTEs occurred in the first 2 weeks of catheter placement versus 6/24 TEs ( $\mathrm{p}=0.001$, chi-square). Catheter survival was longer than 24 weeks in 12/30 catheters. Nine catheters were lost to NTEs. No catheter was lost secondary to a TE. It is therefore concluded that intradialytic outpatient $H D U$ is useful for the treatment of HD catheter thrombosis. It may obviate/reduce the need for catheter stripping or replacement. Randomized controlled trials with this approach are needed. A higher proportion of NTEs tended to occur earlier after catheter placement compared to TEs.

(Hemodial Int, Vol. 4, 32-36, 2000)

\section{Correspondence to:}

Rajiv Saran, MD, MRCP, Division of Nephrology, University of Michigan Medical Center, B-1951, Clinical Faculty Office Building, Box 0704, Ann Arbor, Michigan 48109-0704 U.S.A.

email: rsaran@umich.edu

\section{Key words}

Hemodialysis catheter, malfunction, thrombosis, urokinase, warfarin

\section{Introduction}

A recent editorial review elegantly summarized the importance and optimal use of central venous catheters for hemodialysis (HD) [1]. It highlighted the conundrum the nephrology community faces today. Although it would be desirable to provide native arteriovenous (AV) fistulas to a majority of patients on chronic HD, as outlined in the Dialysis Outcomes Quality Initiative (DOQI) guidelines [2], there will always be a significant proportion of patients for whom a cuffed, tunneled, central venous catheter is the only option, particularly the elderly, diabetics with severe peripheral vascular disease, and patients waiting for AV fistula maturation $[3,4]$. Hence, there is an ongoing need for innovations in design, insertion techniques, and strategies aimed at long-term sustenance of these vital "life-lines."

Although catheters have several advantages, they are beset with two major complications in the form of thrombosis and infection. These complications have had a negative impact on the catheter's potential role as viable long-term vascular access in HD.

Catheter-related thrombosis can broadly be classified as intraluminal (intrinsic) or extraluminal (extrinsic). The latter can be a consequence of trauma to the endothelial lining of the vein and subsequent thrombus formation, or of the frequent development of a fibrin sleeve around the catheter, extending from the point of entry of the catheter into the vein to the catheter tip [5]. It is believed that this phenomenon contributes significantly to the pathogenesis of catheter-related thrombosis.

A wide variety of intraluminal catheter locking strategies have been used. The most popular are the heparin lock and the urokinase lock. More recently, interest has been generated in the concentrated citrate lock [6]. Although successful in the short term, these regimens would, in theory, not be efficacious against an extrinsic clot/fibrin sleeve, and therefore, are likely to have only limited utility in long-term prevention and treatment of catheter-related thrombosis.

Since 1995 at the University of Missouri-Columbia and Dialysis Clinic, Inc. (DCI), catheters malfunctioning due to thrombosis have been successfully treated using an outpatient high-dose urokinase (HDU) infusion in the absence of contraindications to its use. A dose of 250000 IU of urokinase, repeated for three consecutive dialyses, has been successful as well as safe in restoring blood flow to that prescribed in 
99\% of poor flow episodes, as reported in a retrospective analysis by Twardowski [7]. The routine use of intradialytic heparin was continued in all these patients without any bleeding complications. Details of the protocol have been published elsewhere [8].

In this paper, we report a prospective observational study evaluating the use of HDU infusion in the outpatient HD setting to see if this strategy would also be beneficial in prolonging the useful life of HD catheters. In addition, we looked at the role of warfarin as maintenance therapy to prevent catheter thrombosis after the initial use of urokinase.

\section{Patients and methods}

The Institutional Review Board of the University Hospital and Clinics, University of Missouri-Columbia, MO, U.S.A., approved the protocol. Patients were recruited from the DCI outpatient unit between December 1997 and June 1999. These were patients being initiated or maintained on chronic HD with a central venous cuffed catheter as their only blood access route, or awaiting maturation of an AV fistula, commencement of peritoneal dialysis, or living donor transplantation. Informed written consent was obtained from all patients.

\section{Demographic data}

Twenty patients were recruited over the study period. A total of thirty catheters was utilized for these patients. Mean age was $57.7 \pm 13.9$ (SD) years (range 28 - 81 years). There were 13 males and 7 females. Twelve were Caucasian, 7 African American, and 1 Asian. The etiology of end-stage renal disease (ESRD) was diabetes mellitus in 7, hypertension in 8 , and other causes in 5 .

\section{Indications, type of catheter, and site of insertion}

The indications for using a cuffed, tunneled dialysis catheter in these patients are shown in Table I. Sixteen patients had 24 PermCaths (Quinton Instrument Company, Seattle, WA, U.S.A.), 1 patient had a Vas Cath (Bard, Salt Lake City, UT, U.S.A.), 1 patient had 2 Tesio catheters (Medcomp, Harleysville, PA, U.S.A.), 1 patient had an experimental catheter manufactured by Quinton Instrument Company, and 2 patients had Opti-Flow catheters (Bard) placed during the course of this study.

TABLE I Indications for the use of cuffed, tunneled hemodialysis (HD) catheters in this study.

\begin{tabular}{lc}
\hline Indication for HD catheter & Patients (n) \\
\hline Awaiting fistula maturation & 8 \\
Multiple failed arteriovenous accesses & 5 \\
Awaiting peritoneal dialysis & 2 \\
Awaiting living donor transplantation & 2 \\
Steal syndrome & 1 \\
Needle phobia & 1 \\
Patient preference & 1 \\
Total & 20 \\
\hline
\end{tabular}

The sites of insertion were right internal jugular vein (16 catheters), left internal jugular vein (5 catheters), left subclavian vein (7 catheters), and right subclavian vein (2 catheters).

Catheter placement was performed in the operating room by a dedicated team of vascular surgeons. Whether fluoroscopy was consistently used as an aid to catheter placement was not recorded at the time of this data collection. Placement of the catheter tip in the middle of the right atrium is the wellknown best location for ensuring consistently good blood flows on dialysis. We determined catheter tip position by inspection of routine postoperative chest radiographs and measuring (in millimeters) the distance between the catheter tip and the superior vena cava (SVC)-right atrial (RA) junction. If the catheter was in the RA it was recorded as "positive," and if the tip of the catheter was in the SVC or higher it was recorded as "negative." “Zero” position implied tip placement at the RA-SVC junction. The "arterial” and "venous" tip distances from the RA-SVC junction were recorded separately.

\section{Monitoring catheter function}

Long-term catheter survival was defined as continued optimal catheter function for at least 24 weeks (6 months). Follow-up of the study patients was planned for 6 months after catheter insertion or until catheter removal, whichever was earlier. Any complication was recorded, and reasons for catheter removal were documented. All HD sessions were monitored closely. Parameters recorded at half-hourly intervals included blood flow rate (as measured by pump speed), arterial and venous pressures, and systolic and diastolic blood pressure. An "event” was defined as a persistent (continuously, for $\geq 1$ hour during dialysis) drop in blood flow by greater than or equal to $20 \%$ below that prescribed. If catheter position, patient posture, or a hypovolemic episode was found to be the explanation for the drop in blood flow, it was designated a nonthrombotic event (NTE), otherwise it was assumed to be secondary to catheter malfunction due to possible thrombosis, that is, a thrombotic event (TE). In the latter circumstance, in consultation with the patient's nephrologist and having ruled out the presence of any contraindication to its use, the dialysis nurse administered 250000 IU of urokinase, dissolved in $100 \mathrm{~mL}$ of normal saline, over 3 hours via the venous chamber, with continuation of dialysis and the routine intradialytic heparin protocol. The usual protocol at our center stipulates that HDU infusion be readministered during two further consecutive dialysis treatments. It was so planned that if a patient required the use of HDU for two TEs, he/she would be commenced on prophylactic warfarin. International Normalized Ratio (INR) was monitored by weekly predialysis blood sampling. Target INR was $1.0-2.0$, but was regulated upward in a stepwise manner if the patient had further TEs [9].

\section{Statistics}

Descriptive statistics were employed for the most part in this observational study. Fisher's exact test was used to compare 
the incidence of NTE in "negatively"-placed catheters and other catheters, and for comparison of early and late events. A $p$ value of less than 0.05 was considered statistically significant.

\section{Results}

A total of 1239 outpatient dialysis treatments were monitored during this study.

\section{Catheter position data}

Sixteen catheter tips were initially placed in a "negative" position. Of these, 11 sustained NTEs (see below). Determination of the cause of NTE was uncertain, unless the patient had significant hypovolemia and catheter function returned to normal during the next dialysis without intervention other than adjustment of target weight. Most NTEs were believed to be due to poor position of the catheter. Six of the negativelyplaced catheters continued to function satisfactorily for 5 24 weeks; however, 5 of these subsequently had TEs. Only 4 NTEs occurred in the other 14 catheters. Most of these events were believed to be related to hypovolemia. This difference is not significant $(p=0.066)$.

\section{Nonthrombotic events}

There were 15 NTEs in 7 of the 20 patients (mean number of events per affected patient, $2.1 \pm 0.99$; range $1-4$ ). In five instances there was no flow at the outset after initial catheter placement. In one instance of primary nonfunction, the venous limb of a Tesio catheter placed via the left internal jugular vein became kinked in the subcutaneous tissue at the level of the clavicle, and was repositioned successfully via the femoral route by an interventional radiologist. In seven instances, poor blood flow was felt to be due to faulty position of the catheter in the SVC or brachiocephalic vein ("negatively”-placed catheters).

\section{Other catheter complications}

There was one severe exit-site infection requiring catheter removal, and two separate instances of catheters being accidentally cut by the patient: One patient presented to the ER with a cut catheter for which an exact explanation could not be elicited; the catheter had to be replaced. The other patient sustained a cut on his second Tesio catheter 9 weeks following catheter placement while attempting to cut off a dressing with scissors. This was successfully repaired and the catheter continued to function satisfactorily beyond 24 weeks.

\section{Thrombotic events and use of high-dose urokinase}

There were 24 (1.9\%) instances of a drop in blood flow presumed to be due to TE as there was no other obvious explanation. These events occurred in 11 of the 20 patients (mean number of events per affected patient, $2.18 \pm 1.34$; range 1 -5). High-dose urokinase was administered in 22 of these 24 instances (the other two were managed by "catheter stripping” with good results). However, not all TEs were treated with the stipulated three consecutive doses, based on the treating nephrologist's judgement. In 19 TEs at least two consecutive doses of HDU were given ( 9 instances of 2 doses only, 7 instances of 3 doses, and 1 instance each of 4, 5, and 6 consecutive doses of HDU). In the last two instances (i.e., where 5 and 6 doses of HDU were used), there was possible accompanying catheter-related infection that was being treated by intravenous antibiotics.

High-dose urokinase administration was associated with complete recovery of blood flow on dialysis in 21 of the 22 HDU-treated instances of catheter malfunction thought to be due to a TE (95\%). There was partial restoration of blood flow in 1 patient who received only 100000 IU of urokinase instead of $250000 \mathrm{IU}$ at the treating physician's discretion. The pre-HDU blood flows in these 22 instances of catheter malfunction had decreased to a mean of $236.8 \pm 84.5 \mathrm{~mL} / \mathrm{min}$. Post-HDU blood flow increased to a mean of $431 \pm 53.4 \mathrm{~mL} /$ min. This resulted in satisfactory dialysis delivery in all these patients. No side effects such as fever, allergic reactions, or bleeding were observed as a result of HDU administration.

\section{Early versus late events}

Early events were defined as those occurring less than 2 weeks after catheter placement. As depicted in Fig. 1, 12/15 NTEs occurred early versus only 6/24 TEs $(p=0.001)$.

\section{Warfarin data}

Eight of the 11 patients who developed TEs were placed on warfarin post-HDU, with target INR between 1 and 2. Six of these 8 patients had catheter survival of longer than 24 weeks, and the 2 remaining patients had elective removal of their catheter, following maturation of their AV fistulas, at 20 weeks and 14 weeks, respectively.

\section{Catheter survival data}

Causes of catheter loss are shown in Table II. No catheter was lost secondary to a TE during this study. Catheter survival data

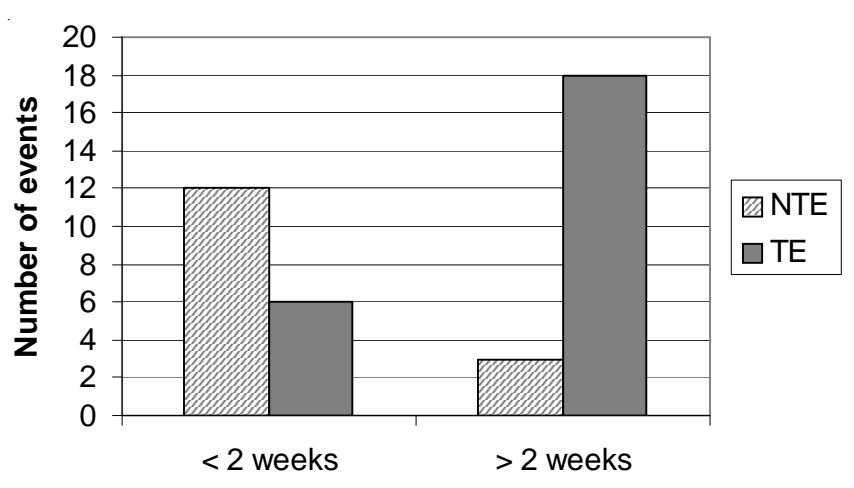

Time after catheter placement

FIGURE 1 Bar graph showing a comparison of early versus late events. Early events were predominantly of the nonthrombotic (NTE) variety. Late events were more often of a thrombotic (TE) nature. 
are shown in Fig. 2. Thirteen catheters were lost between 0 and 10 weeks ( 9 due to faulty initial positioning, 3 were elective removals at access maturation, and 1 was lost to follow-up). Twelve of 30 catheters survived more than 24 weeks.

\section{Discussion}

Low-dose intraluminal urokinase locks (5000 - 9000 IU/ lumen of catheter) have been in use since the late1980s and have been reported to result in success rates of $74 \%-95 \%$ in keeping dialysis catheters patent $[10,11]$. Experience at the University of Missouri with long-term catheters indicated that, after several months of catheter use, such locks had only a $40 \%-50 \%$ success rate [12]. The urokinase locks sometimes have to be repeated several times [13] and result in time waste in the outpatient dialysis unit. This not only increases cost of care, but also compromises time on dialysis. In addition, this strategy does not ensure long-term catheter survival.

Intraluminal-locking strategies are not useful in the prevention or treatment of "extrinsic" thrombosis. They are directed at intraluminal thrombi and cannot be expected to have any major impact on fibrin sleeves or intramural thrombi

TABLE II Causes of catheter loss during the course of this study. No catheter was lost to a thrombotic event.

\begin{tabular}{lc}
\hline Reasons for catheter loss & Catheters [n (\%)] \\
\hline Nonthrombotic event & $9(30)$ \\
Elective removal & $3(10)$ \\
Exit-site infection & $1(3)$ \\
Catheter cut accidentally & $1(3)$ \\
Patient lost to follow-up & $3(10)$ \\
Death & $1(3)$ \\
Thrombotic event & $0(0)$ \\
Total number of catheters & 30 \\
\hline
\end{tabular}

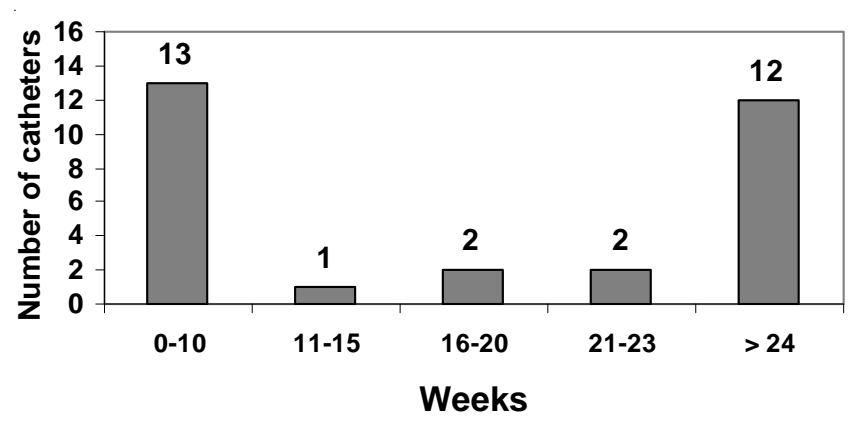

FIGURE 2 Bar chart showing the length of follow-up of patients with cuffed tunneled catheters in this study. Patients were followed for a maximum of 24 weeks or loss of catheter, whichever was earlier. Between 0 and 10 weeks, 13 catheters were lost (9 secondary to nonthrombotic events, 3 to elective removal, and 1 lost to follow-up). Between 11 and 15 weeks, 1 catheter was electively removed. Of the 2 catheters lost between 16 and 20 weeks, 1 patient was lost to follow-up and the other died. One elective removal and 1 loss to follow-up accounted for 2 catheters that survived between 21 and 23 weeks. adhering to the venous wall. Although it is important to keep the lumen of a dialysis catheter clot-free at all times by using an effective locking system, it is vital to develop strategies that will prevent or correct the abnormalities around the catheter that predispose to thrombus formation. Strategies aimed at retarding or eliminating the formation of a fibrin sleeve could potentially enhance catheter survival.

We believe that, although not preventative, systemic urokinase infusion is beneficial for attenuating extrinsic clots and perhaps the fibrin sleeve, although our study does not directly prove this point as no contrast imaging procedure was used before or after HDU infusion. If this is combined with maintenance therapy with warfarin, we feel that longterm survival of permanent dialysis catheters can be improved in patients who are predisposed to thrombotic complications.

To the best of our knowledge, this study is the first prospective evaluation of outpatient use of HDU infusions in the management of cuffed dialysis catheters. There have been three prior retrospective studies [14-16] looking at this issue.

Our study is not confirmatory owing to its observational design. The number of patients who received warfarin after having two or more TEs treated by HDU, versus those that did not, is not sufficient to arrive at any definitive conclusions about a potential prophylactic effect of warfarin. The effectiveness of low-dose warfarin in preventing thrombotic complications in infusion catheters has been determined in several studies [17-19]. There are only a few retrospective studies on the effectiveness of stepwise dosing of warfarin for HD catheters $[7,9,20]$. Therefore, it would be desirable to conduct a randomized, controlled clinical trial using the approach of HDU followed by warfarin in patients who develop catheter-related thrombosis. Future studies may evaluate the optimal dose of urokinase, the number of infusions, and the ideal INR range to prevent subsequent TEs.

In this uncontrolled, prospective observational study, we confirmed our earlier observation in HD patients that HDU does indeed improve suboptimal blood flows related to thrombosis. In addition, we confirmed the safety of HDU, as we did not encounter any complication related to its use. Of course, it is imperative to systematically rule out both absolute and relative contraindications for the use of a thrombolytic agent prior to its use.

Another potential advantage to HDU is the relatively low cost. Although we have not performed an updated cost analysis, data have been published [8] showing HDU to be considerably cheaper than either catheter stripping or surgical replacement of a dialysis catheter at our center. Toward the latter third of the study period, fresh supplies of urokinase became unavailable and this study could not be continued. However, we continued to use remaining supplies of urokinase until June 1999.

Urokinase is not available at present and it is not known when it will again become available. Although tissue plasminogen activator (tPA) is much more expensive than 
urokinase, it may be the preferred fibrinolytic agent as it binds to the clot, activates plasminogen in situ, and causes fibrinolysis not only on the surface of a thrombus but also within the thrombus [21]. In a study comparing the effectiveness of a 2-mg lock with tPA to 10000 IU of urokinase, tPA was more effective [22]. Infusions of higher doses of tPA are theoretically possible, and a systematic study of this agent may be warranted despite the higher cost.

\section{Acknowledgments}

We are grateful to Dialysis Clinic, Inc. for funding this study. We are especially indebted to the hemodialysis nursing staff at DCI, Columbia, Missouri, for carrying out the high-dose urokinase protocol so efficiently.

\section{References}

1 Schwab SJ, Beathard G. The hemodialysis catheter conundrum: Hate living with them, but can't live without them. Kidney Int. 56:1-17, 1999.

2 Schwab S, Besarab A, Beathard G, Brouwer D, Etheredge E, Hartigan M, Levine M, McCann R, Sherman R, Trerotola S. National Kidney Foundation - Dialysis Outcomes Quality Initiative clinical practice guidelines for vascular access. Guideline 3: Selection of permanent vascular access and order of preference for placement of AV fistulae. Am J Kidney Dis. 30(suppl 3):S156-7, 1997.

3 Riordan S, Frawley J, Gray L, Niesche J. Primary access surgery for long-term hemodialysis. Aust N Z J Surg. 64:763-7, 1994.

4 Windus DW, Jendrisak MD, Delmez JA. Prosthetic fistula survival and complications in hemodialysis patients: Effect of diabetes and age. Am J Kidney Dis. 19:448-52, 1992.

5 Hoshal VL Jr, Ause RG, Hoskins PA. Fibrin sleeve formation on indwelling subclavian central venous catheters. Arch Surg. 102:253-8, 1971.

6 Ash SR, Mankus RA, Sutton JM, Criswell RE, Crull RC, White LE, Lowrey SJ. Concentrated citrate for catheter lock solution (Abstract). J Am Soc Nephrol. 10:272A, 1999.

7 Twardowski ZJ. High-dose intradialytic urokinase to restore patency of permanent central vein hemodialysis catheters. Am J Kidney Dis. 31:841-7, 1998.

8 Twardowski ZJ. The clotted central venous catheter for haemodialysis. Nephrol Dial Transplant. 13:2203-6, 1998.

9 Twardowski Z. Stepwise anticoagulation with warfarin for prevention of intravenous catheter thrombosis. Hemodial Int. 4:37-41, 2000.

10 Seddon PA, Hrinya MK, Gaynord MA, Lion CM, Mangold
BM, Bruns FJ. Effectiveness of low dose urokinase on dialysis catheter thrombolysis. ASAIO J. 44:M559-M561, 1998.

11 Schwab SJ, Buller GL, McCann RL, Bollinger RR, Stickel DL. Prospective evaluation of a Dacron cuffed hemodialysis catheter for prolonged use. Am J Kidney Dis. 11:166-9, 1988.

12 Twardowski ZJ. Percutaneous blood access for hemodialysis. Semin Dial. 8:175-86, 1995.

13 Bour ES, Weaver AS, Yang HC, Gifford RR. Experience with the double lumen Silastic catheter for hemoaccess. Surg Gynecol Obstet. 171:33-9, 1990.

14 Uldall R, Besley ME, Thomas A, Salter S, Nuezca LA, Vas M. Maintaining the patency of double-lumen silastic jugular catheters for hemodialysis. Int J Artif Organs. 16:37-40, 1993.

15 Lund GB, Trerotola SO, Scheel PF Jr, Savader SJ, Mitchell SE, Venbrux AC, Osterman FA Jr. Outcome of tunneled hemodialysis catheters placed by radiologists. Radiology. 198:467-72, 1996.

16 Trerotola SO, Johnson MS, Harris VJ, Shah H, Ambrosius WT, McKusky MA, Kraus MA. Outcome of tunneled hemodialysis catheters placed via the right internal jugular vein by interventional radiologists. Radiology. 203:489-95, 1997.

17 Bern MM, Lokich JJ, Wallach SR, Bothe A Jr, Benotti PN, Arkin CF, Greco FA, Huberman M, Moore C. Very low doses of warfarin can prevent thrombosis in central venous catheters. A randomized prospective trial. Ann Intern Med. 112:423-8, 1990.

18 Boraks P, Seale J, Price J, Bass G, Ethell M, Keeling D, Mahendra P, Baglin T, Marcus R. Prevention of central venous catheter associated thrombosis using minidose warfarin in patients with haematological malignancies. Br J Haematol. 101:483-6, 1998.

19 Veerabagu MP, Tuttle-Newhall J, Maliakkal R, Champagne C, Mascioli EA. Warfarin and reduced central venous thrombosis in home total parenteral nutrition patients. Nutrition. 11:142-4, 1995.

20 Pierratos A. Nocturnal home hemodialysis: An update on a 5-year experience. Nephrol Dial Transplant. 14:2835-40, 1999.

21 Davenport A. Central venous catheters for hemodialysis: How to overcome the problems. Hemodial Int. 4:78-82, 2000.

22 Atkinson JB, Bagnall HA, Gomperts E. Investigational use of tissue plasminogen activator (t-PA) for occluded central venous catheters. JPEN J Parenter Enteral Nutr. 14:310-11, 1990. 